\title{
Mass effect on the lithium abundance evolution of open clusters: Hyades, NGC 752, and M 67
}

\author{
M. Castro ${ }^{1}$, T. Duarte ${ }^{1}$, G. Pace ${ }^{2}$, and J.-D. do Nascimento Jr. ${ }^{1,3}$ \\ ${ }^{1}$ Departamento de Física Teórica e Experimental, Universidade Federal do Rio Grande do Norte, 59072-970 Natal, RN, Brazil \\ e-mail: mcastro@dfte.ufrn.br \\ 2 Instituto de Astrofísica e Ciência do Espaço, Universidade do Porto, Rua das Estrelas, 4150-762 Porto, Portugal \\ ${ }^{3}$ Harvard-Smithsonian Center for Astrophysics, Cambridge, MA 02138, USA
}

Received 16 October 2015 / Accepted 12 March 2016

\begin{abstract}
Lithium abundances in open clusters provide an effective way of probing mixing processes in the interior of solar-type stars and convection is not the only mixing mechanism at work. To understand which mixing mechanisms are occurring in low-mass stars, we test non-standard models, which were calibrated using the Sun, with observations of three open clusters of different ages, the Hyades, NGC 752, and M67. We collected all available data, and for the open cluster NGC 752, we redetermine the equivalent widths and the lithium abundances. Two sets of evolutionary models were computed, one grid of only standard models with microscopic diffusion and one grid with rotation-induced mixing, at metallicity $[\mathrm{Fe} / \mathrm{H}]=0.13,0.0$, and $0.01 \mathrm{dex}$, respectively, using the Toulouse-Geneva evolution code. We compare observations with models in a color-magnitude diagram for each cluster to infer a cluster age and a stellar mass for each cluster member. Then, for each cluster we analyze the lithium abundance of each star as a function of mass. The data for the open clusters Hyades, NGC 752, and M 67, are compatible with lithium abundance being a function of both age and mass for stars in these clusters. Our models with meridional circulation qualitatively reproduce the general trend of lithium abundance evolution as a function of stellar mass in all three clusters. This study points out the importance of mass dependence in the evolution of lithium abundance as a function of age. Comparison between models with and without rotation-induced mixing shows that the inclusion of meridional circulation is essential to account for lithium depletion in low-mass stars. However, our results suggest that other mechanisms should be included to explain the Li-dip and the lithium dispersion in low-mass stars.
\end{abstract}

Key words. stars: fundamental parameters - stars: abundances - stars: evolution - stars: interiors - stars: solar-type

\section{Introduction}

Lithium $\left({ }^{7} \mathrm{Li}\right)$ is a fragile element that is destroyed at temperatures above $\sim 2.5 \times 10^{6} \mathrm{~K}$. The lithium depletion in stars depends on several factors such as mass, age, metallicity, rotation, magnetic fields, mass loss, and mixing mechanisms (e.g., D’Antona \& Mazzitelli 1984; Deliyannis \& Pinsonnault 1997; Ventura et al. 1998; Charbonnel \& Talon 2005). Abundance measurements of this element in open cluster stars allow us to investigate mixing in stellar interior and to put constraints on the non-convective mixing processes as a function of mass, age, rotation, and metallicity for coeval stars. To understand the mixing mechanisms and to explain the complex evolution of stellar chemical elements, we need to constrain the effect of mass. As a part of this complexity, several unexpected results related to the depletion of lithium in stars have been found and reveal our limited understanding of the physics acting in the stars' interiors. One of these unexpected results concerns the measurements of lithium abundance in late-F and early G-type stars, showing empirical evidence that is in contradiction with the standard model predictions, which only include convection. For stars in these spectral classes, authors have shown lithium depletion during the main sequence (Boesgaard \& Tripicco 1986; Boesgaard 1987; Pilachowski \& Hobbs 1988). However, convective zones of low-mass main sequence (MS) stars do not reach regions in the stellar interior, where the temperature is sufficiently high to drive lithium destruction (see e.g., Randich et al. 2006, and references therein). This depletion is mass-age dependent (do Nascimento et al. 2009; Meléndez et al. 2010; Pace et al. 2012). For stars of one solar mass, including the Sun and those stars, which are spectroscopically indistinguishable from the Sun called solar twins (Cayrel de Strobel 1996), the extremely low lithium abundance of about one hundred times lower than its original value as measured in meteorites, represents a longstanding puzzle.

During the pre-main-sequence (PMS), studies showed that $\mathrm{Li}$ destruction in stars with mass that is equal or larger to the solar one should be weak (Martin et al. 1993; Jones et al. 1997). Randich et al. (1997) determined the lithium abundance in 28 stars of the 28 Myr old cluster IC 2602. They compared the lithium abundance distribution as a function of stellar mass of this sample with those of the $\alpha$ Persei (50 Myr old) and the Pleiades (70 Myr old) clusters, and showed that only latest-type stars present a significant lithium depletion during the PMS. Early-K and G-type stars have a slightly lower lithium abundance in the oldest clusters, and F-type stars do not present any significant lithium depletion.

According to the standard stellar models, lithium depletion should be a unique function of mass, age, and chemical composition. Stars with close effective temperature in a given cluster should have undergone the same amount of lithium depletion. In this context, lithium abundances have been studied extensively in open clusters of different ages and revealed a complex behavior with an important scattering (see e.g., 
Pinsonneault 1997, and references therein). The star-to-star scatter in lithium abundance exists among solar-type stars of the 2 Gyr old cluster NGC 752, as well as in the solar age, solar metallicity open cluster M67 (e.g., Pasquini et al. 1997; Jones et al. 1999). From the study of the open cluster NGC 752 by Hobbs \& Pilachowski (1986a), Pilachowski \& Hobbs (1988), and Balachandran (1995), and from the 4 Gyr old open cluster M 67 studied by Hobbs \& Pilachowski (1986b), Spite et al. (1987), and Garcia Lopez et al. (1988), authors conclude about a significant depletion relative to younger systems that increases with age. Despite decades of observations in clusters and field stars, the mechanism that drives the evolution of the surface lithium abundance and its scattering in main-sequence field stars and stars belonging to open clusters is far from being fully understood, and the lithium depletion expected from theoretical models needs to be adjusted to reproduce the mass and agedependence. As for the open cluster M 67 (Pace et al. 2012), we explore this mass-age dependence and the star-to-star scatter at the same mass and different ages, with particular attention to the quality of the spectroscopy behind the lithium abundance determination. In this work, we continue to homogenize previous studies on open clusters by obtaining a consistent set of temperatures and masses, and by applying corrections to the lithium abundances when needed, due to revised effective temperatures, by taking into account non-local thermodynamic equilibrium (LTE) effects on the lithium abundances. We analyze stars from the main-sequence to the red giant branch, comparing our models to the observed behavior of lithium abundance as a function of mass and age.

This paper is organized as follows. In Sect. 2, we describe the working sample of the three clusters. In Sect. 3, we present details of our evolutionary stellar models, and in Sect. 4 we construct the color-magnitude diagrams (CMD) of the open clusters. In Sect. 5, we discuss the evolution of the lithium abundance predicted from our models. In Sect. 6, we compare the models with the observations from the studied clusters. Finally, in Sects. 7 and 8, we present our discussion and conclusions.

\section{Working sample}

Our working sample is composed of lithium non-LTE abundance measurements for 67 F-G dwarfs in the Hyades from Takeda et al. (2013). Takeda et al. re-studied 49 stars with lithium abundance measurements that are available in the literature (Randich et al. 2007; Thorburn et al. 1993; Soderblom et al. 1990; Boesgaard \& Budge 1988; Boesgaard \& Tripicco 1986; Duncan \& Jones 1983; Rebolo \& Beckman 1988), and added 19 more stars to their sample. This sample is currently the largest for lithium abundances of Hyades stars. We decided not to use the star HD 285252 of the original sample because its lithium abundance is very low and uncertain $(A(\mathrm{Li})=-0.29)$. Takeda et al. (2013) do not directly provide uncertainty in their lithium abundance measurements, but they do provide all necessary data in electronic format to compute it. The uncertainty on lithium abundance reported in Table A.1 was obtained by adding its three components quadraticall, i.e., that originated from the error of temperature, gravity, and microturbolence, respectively. Each term was defined as $1 / 2(A(\mathrm{Li}, P-\Delta P)-A(\mathrm{Li}, P+\Delta P))$, where $P$ is the parameter considered (temperature, gravity, or microturbulence), $\Delta P$ its uncertainty according to Takeda et al. (2013), which assumes $\Delta T=100 \mathrm{~K}$, and the two other parameters were kept constant. The temperature is the only significant source of error for the lithium abundance.
Masses and stellar parameters of the 67 sample stars were computed for each star by taking the closest isochrone point to the stellar data-point on the color-magnitude diagram (see Sect. 4). The isochrone was chosen by imposing $[\mathrm{Fe} / \mathrm{H}]=0.13$ (Paulson et al. 2003) and selecting the best fitting age, which was found to be 0.79 Gyr. $B-V$ colors and absolute $V$ magnitude are taken from Takeda et al. (2013), who in turn used photometry and parallaxes for individual Hyades members from the HIPPARCos catalogue (de Bruijne et al. 2001; Perryman 1997). Lithium abundances for the individual Hyades stars are directly derived by Takeda et al. (2013), and do not take into account this adjustment on the stellar parameters, which was applied by projection on the isochrone.

Takeda's lithium abundance measurements are already accurate and homogeneous. Revisiting them, as we did for NGC752 stars, would not change the conclusions of this paper, so we did not. The calculation of stellar parameters for Hyades stars was mainly intended to derive stellar masses, which we chose as an independent variable to examine the trend of lithium abundances, as we did for the other clusters.

Equivalent width (EW) measurements of the lithium line at $6708 \AA$ for NGC 752 members were taken from the following sources: Sestito et al. (2004), Hobbs \& Pilachowski (1986a), Pilachowski \& Hobbs (1988). Pilachowski et al. (1988) give lithium abundances (mostly upper limits) for 11 giants, which were transformed into (upper limits of) EWs by reverting the LTE analysis using the Lind et al. (2009) code. Since Pilachowski et al. do not mention what metallicty they adopted, we assumed a solar metallicity for the stars. There are three stars in common between the older works and that of Sestito et al. In these cases, the value from the latter work is adopted. The mean difference between the EWs that is published in the two works for these three stars in common is used as an estimation of the errors on the EW for the other measurements given in Hobbs \& Pilachowski (1986a), because they are not provided by the authors. The stars in common are PLA701, PLA859, and PLA921 (H146, H207, and H229, respectively, in the numbering system by Heinemann 1926), and the couple of measurements are, respectively, 42 and 45, 26.2 and 7, and 54 and 63. The values are in $\mathrm{m} \AA$, the result from Sestito et al. being the first of each pair. The average difference, after rounding, is $10 \mathrm{~m} \AA$, which we adopted as error on EW for all stars for which only the old measurements are available. The EWs collected were used to compute abundances with the code by K. Lind, employing the full non-LTE analysis and the stellar parameters computed, as described below.

Errors on lithium abundances were calculated by adding quadratically its two main components, i.e., that due to the equivalent width and that due to the temperature. For the former, we simply computed: $\mathrm{d} A(\mathrm{Li})_{\mathrm{EW}}=1 / 2\left(A\left(\mathrm{Li}, E W+\Delta_{\mathrm{EW}}\right)-\right.$ $\left.A\left(\mathrm{Li}, E W-\Delta_{\mathrm{EW}}\right)\right)$, where temperature, gravity, and metallicity were kept constant. To estimate the error due to the uncertainty in the temperature, we computed each stellar temperature in two different ways. The most accurate value was drawn from the isochrone in the CMD, as described in more detail below. We will refer to it as $T_{\text {iso }}$. The other value, $T_{\text {phot }}$, was obtained from the photometric calibration of Casagrande et al. (2010), or Kučinskas et al. (2005) for the giants, and the photometry in the reference papers. We then compute, for each dwarf, the following: $\mathrm{d} A(\mathrm{Li})_{\mathrm{T}}=A\left(\mathrm{Li}, T_{\text {iso }}\right)-A\left(\mathrm{Li}, T_{\text {phot }}\right)$.

The mean cluster iron content estimates range from $[\mathrm{Fe} / \mathrm{H}]=$ -0.09 dex (Hobbs \& Thorburn 1992, based on 8 dwarfs) to $[\mathrm{Fe} / \mathrm{H}]=0.08 \pm 0.04$ (Carrera \& Pancino 2011, based on 
M. Castro et al.: Mass effect on the lithium abundance evolution of open clusters

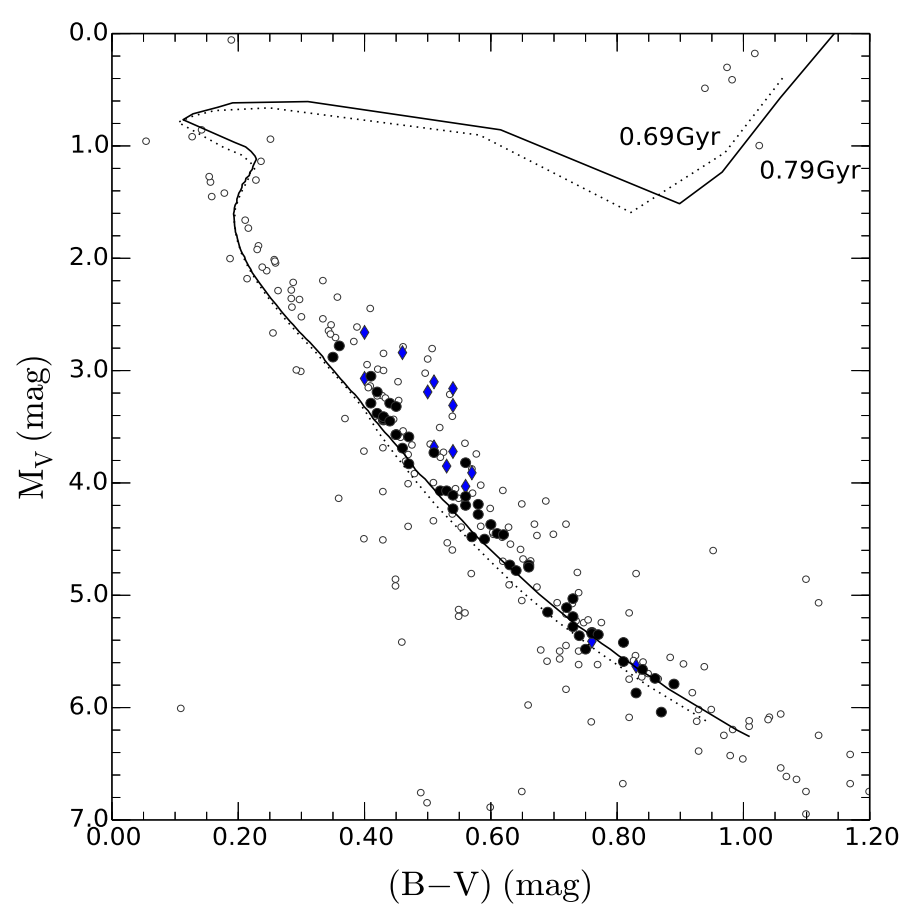

Fig. 1. Color-magnitude diagram of the Hyades open cluster. Open circles are the whole sample of Hyades stars with magnitudes from the HIPPARCOS catalogue. Filled black circles are the sample of stars form Takeda et al. (2013) for which we have a lithium abundance estimation. Blue diamonds represent the known binaries of this sample. The continuous line is the isochrone of $0.79 \mathrm{Gyr}$, calculated from our TGEC models with extra mixing, and the dotted line is the isochrone of $0.69 \mathrm{Gyr}$, calculated from standard models.

4 giants). The other two studies (Sestito et al. 2004, based on 18 dwarfs, and Reddy et al. 2012, based on 4 giants) favour a substantially solar metallicity $([\mathrm{Fe} / \mathrm{H}]=0.01 \pm 0.04$ and $[\mathrm{Fe} / \mathrm{H}]=-0.02 \pm 0.05$, respectively). In conclusion, the range of possible values for the cluster metallicity is approximately $[\mathrm{Fe} / \mathrm{H}]=0.0 \pm 0.1$.

As we did for the Hyades, we computed stellar parameters for each star (effective temperature and mass) from the isochrone point closest to the stellar data point on the color-magnitude diagram. We adopted the best fitting isochrone, with an age of $1.59 \mathrm{Gyr}$ for $[\mathrm{Fe} / \mathrm{H}]=0.0$ (see Sect. 4 ).

The stars of both samples of the Hyades and NGC 752 clusters were all supposed to be single stars when included in the reference studies. When we checked the data using the study of Van Leeuwen (2007), queried through WEBDA, it turned out that these samples were contaminated by binaries. The contamination is about $20 \%$ for the Hyades cluster (14 out of 67) and for NGC 752 (10 out of 46). All known binaries in both samples are identified in Tables A.1 and A.2 and in Figs. 1, 2, 6, and 7.

The data relative to lithium abundance for the M 67 cluster, corrected for non-LTE effects using the grid of Lind et al. (2009), were analyzed in Pace et al. (2012), to whom we redirect the reader.

\section{Stellar evolutionary models}

We present here the models computed using the ToulouseGeneva stellar evolution code (TGEC; Hui-Bon-Hoa 2008; do Nascimento et al. 2009). We used the same input physics as in Pace et al. (2012). In particular, the transport of chemical

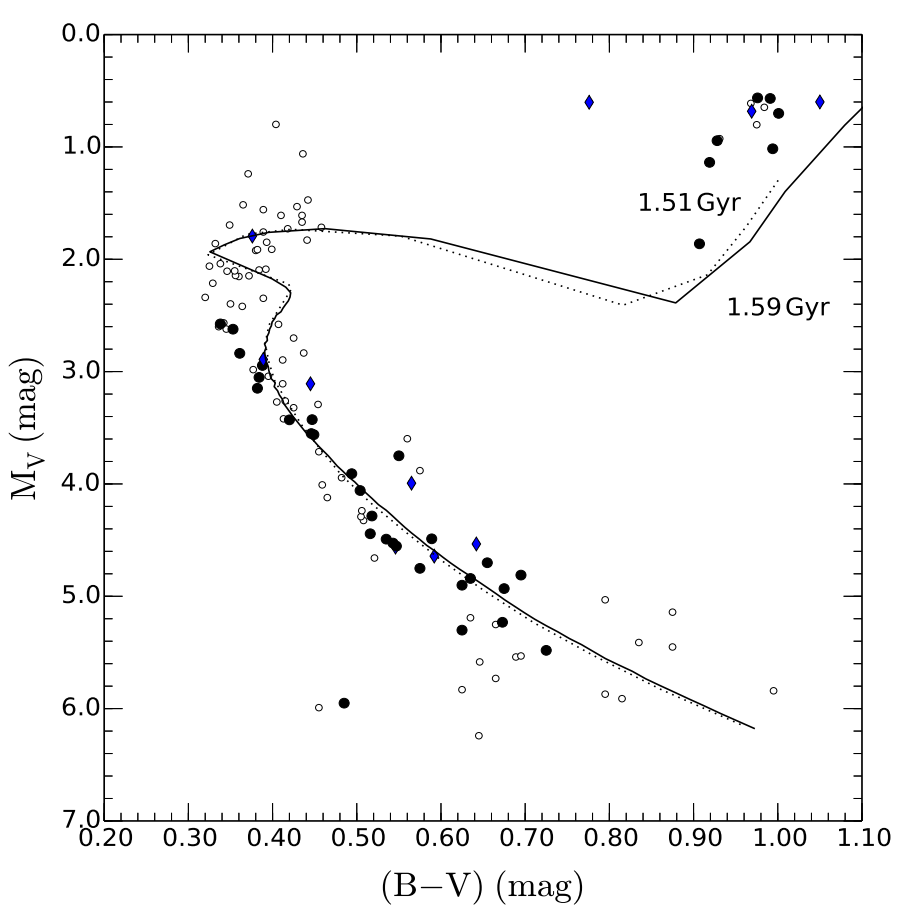

Fig. 2. Color-magnitude diagram of the open cluster NGC 752. Open circles are the whole sample of NGC 752 stars from Daniel et al. (1994). Filled black circles are our working sample of stars for which we have a lithium abundance estimation. Blue diamonds represent the known binaries of this sample. The continuous line is the isochrone of $1.59 \mathrm{Gyr}$ calculated from our TGEC models with extra mixing and the dotted line is the isochrone of $1.51 \mathrm{Gyr}$ calculated from standard models.

elements and angular momentum is driven by different processes, which we briefly describe hereafter.

In the outer layers, convection is modeled according to the Böhm-Vitense (1958) formalism of the mixing-length theory. The mixing-length parameter $\alpha=l / H_{\mathrm{p}}$, where $l$ is the characteristic mixing length and $H_{\mathrm{p}}$ is the pressure scale height, is a free parameter in our models. Below the convective zone, we introduce a convective undershooting with a depth of $0.09 H_{\mathrm{p}}$ so that, for the Sun, the combined mixing reaches the depth deduced by helioseismology $\left(r_{\mathrm{cz}} / R_{\odot}=0.713 \pm 0.001\right.$, Basu \& Antia 1997).

In the radiative zone, microscopic diffusion, which is the process of element segregation by gravitational and thermal diffusion (Eddington 1916; Chapman 1917), is treated by computing convergent series of a Maxwellian distribution function, which is the solution at equilibrium of the Bolzmann equation for dilute collision-dominated plasmas, with sucessive approximations (see the Chapman-Enskog procedure described in Chapman \& Cowling 1970). For collisons between charged ions, we use the Paquette et al. (1986) method, which introduces a screened coulomb potential. Microscopic diffusion is essential in stellar models to account for abundances anomalies in Ap and Am stars (Michaud 1970) and to improve the consistency with helioseismology (Cox et al. 1989; Bahcall et al. 1995; Christensen-Dalsgaard et al. 1996; Richard et al. 1996; Turcotte et al. 1998; Schlattl 2002).

When a star rotates, centrifugal effects modify the gravity equipotentials, which are no longer spherical. It induces a macroscopic circulation of matter in the radiative zone between polar and equatorial regions, the so-called meridional circulation (Eddington 1926; Sweet 1950). Zahn (1992) suggested that the meridional flow induces a transport of angular momentum, creating shears which become unstable in the horizontal 
direction, while the vertical shears are stabilized by the density gradient. The coupling between the meridional advection and the horizontal turbulence leads to an anisotropic mixing of the chemical species, parameterized as an effective diffusion coefficient. Mestel \& Moss (1986) showed that the nuclear-induced $\mu$-gradients slowly stabilize the circulation and expel it from the core toward the external layers. Vauclair \& Théado (2003) show that this feedback effect, which is due to the $\mu$-gradients, strongly modifies the meridional circulation and meanwhile reduces the efficiency of diffusion. Following Zahn (1992), Talon \& Zahn (1997), and Maeder \& Zahn (1998), the rotational mixing is computed by a diffusion coefficient that we write as

$D_{\text {turb }}=\alpha_{\text {turb }} r\left|U_{\mathrm{r}}\right|$

with

$\alpha_{\mathrm{turb}}=C_{\mathrm{v}}+\frac{1}{30 C_{\mathrm{h}}}$

where $C_{\mathrm{v}}$ and $C_{\mathrm{h}}$ are unknown parameters relating to the vertical and horizontal part of the shear-induced anisotropic turbulent coefficient that is due to the transport of angular momentum, respectively. According to the assumption of strong anisotropic turbulence, they must satisfy the condition: $C_{\mathrm{v}} \ll C_{\mathrm{h}} . \boldsymbol{U}_{\mathrm{r}}$ is the vertical velocity amplitude which includes a classical meridional circulation term, which is directly linked to the rotation velocity $\Omega$, a $\mu$-gradient-induced term, a term related to the time variations of the differential rotation, and a term that appears in the case of large horizontal turbulence (see Théado \& Vauclair 2003, for more details).

At the bottom of the convective zone, the transition layer between the differential rotation of the convective zone and the radiative interior rotating as a solid body is called the tachocline. This shear layer undergoes a strong anisotropic turbulence, with much stronger viscous transport in the horizontal than in the vertical direction, reducing the differential rotation and inhibiting its spread deep inside the radiative interior (Spiegel \& Zahn 1992). We assume that the absolute size of the tachocline is constant with time. Brun et al. (1998) showed it can be modeled by an exponential diffusion coefficient added to the turbulent diffusion coefficient. Thus, we use an effective diffusion coefficient:

$D_{\text {eff }}=D_{\text {turb }}+D_{\text {tacho }}$

with

$D_{\text {tacho }}=D_{\text {bcz }} \exp \left(\ln 2 \cdot \frac{r-r_{\text {bcz }}}{\Delta}\right)$

where $D_{\mathrm{bcz}}$ and $r_{\mathrm{bcz}}$ are, respectively, the diffusion coefficient and the radius at the base of the convective zone, and $\Delta$ is the half width of the tachocline (Richard et al. 2004). This is the effective diffusion coefficient that appears in the equation of the chemical transport for the mean concentration of the different species.

We calibrated our models with the Sun as in Richard et al. (2004). There is no a priori reason why the calibration of the parameters for the model of $1.00 M_{\odot}$ should also hold for different masses and evolutionary times but, given that no other standard calibrators are available, our approach is the best possible one for now. The calibration procedure of our models is the same as described in Sect. 4.2 in Pace et al. (2012). The mixing-length parameter $\alpha$ and the initial helium abundance $Y_{\text {ini }}$ enable us to calibrate the present evolutionary status of the Sun. By triangulation, we found the best couple $\left(\alpha, Y_{\text {ini }}\right)$ that allows us to reproduce the solar radius and the solar luminosity at the solar age $\left(L_{\odot}=3.8515 \pm 0.0055 \times 10^{33} \mathrm{erg} \mathrm{s}^{-1}\right.$ and
$R_{\odot}=6.95749 \pm 0.00241 \times 10^{10} \mathrm{~cm}$ at $t_{\odot}=4.57 \pm 0.02 \mathrm{Gyr}$ Richard et al. 2004). For the best-fit solar model, we obtained $L=3.8501 \times 10^{33} \mathrm{erg} \mathrm{s}^{-1}$ and $R=6.95524 \times 10^{10} \mathrm{~cm}$ at an age $t=4.57$ Gyr with $\alpha=1.69$ and $Y_{\text {ini }}=0.268$. The rotationinduced mixing efficiency (meridional circulation and turbulent motions) is calibrated using the parameters $C_{\mathrm{h}}$ and $\alpha_{\text {turb }} . C_{\mathrm{v}}$ is determined from these two parameters through Eq. (2). As explained in Richard et al. (2004), the feedback currents, which are due to $\mu$-gradients, are strongly dependent on $C_{\mathrm{h}}$, which is directly related to the horizontal turbulence. A strong horizontal turbulence tends to homogenize the horizontal layers and thereby smooths the horizontal $\mu$-gradients. In this case, the small induced $\mu$-currents may not be able to compensate for the currents because of classical meridional circulation and the mixing may remain efficient during a long timescale. In particular, we intend to put a mixing that is efficient and deep enough to smooth the diffusion-induced helium gradient, which lies below the surface convective zone, thus improving the agreement between the model and seismic sound-speed profiles. On the other hand, weak horizontal turbulence leads to important $\mu$-currents, which strongly reduce the mixing. In our models, this had to be weak and shallow enough to avoid the destruction of beryllium. We used the values $C_{\mathrm{h}}=9000$ and $\alpha_{\text {turb }}=1$, and obtained an excellent agreement with helioseismology, which was more accurate than $1 \%$ for most of the stars in terms of sound velocity, except in the deep interior, where the discrepancy reaches $1.5 \%$. We also obtained a slight destruction of beryllium by a factor of 1.17 with respect to the meteoritic value, which is well within the error in the determination of the solar beryllium abundance following Grevesse \& Sauval (1998). Beryllium destruction in low-mass stars will be subject of a further study. Finally, the tachocline is calibrated using the parameters $D_{\text {bcz }}$ and $\Delta$ to obtain the solar lithium abundance at the solar age. Helioseismic constraints indicate that the solar tachocline thickness, defined as the region where the effective diffusion coefficient $D_{\text {tacho }}$ increases from 0.08 to 0.92 of its maximum value $D_{\text {bcz }}$, should be lower than $0.04 R_{\odot}$ (Charbonneau at al. 1999), leading to a $\Delta$ lower than $0.01135 R_{\odot}$ (Richard et al. 2004). Using $D_{\text {bzc }}=2.02 \times 10^{5} \mathrm{~m}^{2} \mathrm{~s}^{-1}$ and $\Delta=0.60 \times 10^{9} \mathrm{~cm}=0.0086 R_{\odot}$, we obtained a surface lithium content relative to the initial one $\mathrm{Li} / \mathrm{Li}_{0}=0.60 \times 10^{-2}$. To obtain the lithium abundance $A(\mathrm{Li})$, the question of the initial abundance on the ZAMS is important. Stars with different masses in the range 0.8-2.0 $M_{\odot}$ do not suffer the same lithium depletion during the PMS. In the Sun, this depletion should have been low and, therefore, we kept following the calibration procedure from Richard et al. (1996, 2004), which neglects the lithium destruction during the PMS in solar models. Consequently, we adopted the meteoritic lithium abundance from Asplund et al. (2009) $A(\mathrm{Li})_{0}=3.26$ for the initial abundance, and obtained at the solar age $A(\mathrm{Li})=1.04$, which agrees well with solar lithium abundance measurements from Asplund et al. (2009) $(A(\mathrm{Li})=1.05 \pm 0.10)$.

The three steps of the calibration are not completely independent as the variations in the rotation-induced mixing parameters slightly modify the luminosity and radius of the model. Some iterations are necessary to obtain the best-fit solar model.

We computed two grids of evolutionary models for each cluster, one grid of rotation-induced mixing, and another grid of standard models (with microscopic diffusion), to check the influence of the rotation-induced mixing on the lithium abundance depletion. For the Hyades, we computed models with a range of masses from 0.80 to $2.25 M_{\odot}$ with a step of $0.01 M_{\odot}$ and with a metallicity of $[\mathrm{Fe} / \mathrm{H}]=0.13$. For NGC 752, we computed models with masses from 0.80 to $1.77 M_{\odot}$ with a step of 
$0.01 M_{\odot}$ with a metallicity of $[\mathrm{Fe} / \mathrm{H}]=0.0$. Finally, for M 67 , our models have been computed with masses in the range 0.90 to $1.34 M_{\odot}$, with a step of $0.01 M_{\odot}$, and with a metallicity of $[\mathrm{Fe} / \mathrm{H}]=0.01$, which is a simple average of different estimates for the metallicity of M67 (see Pasquini et al. 2008). We ran the models from the zero age main sequence (ZAMS) to the top of the red giant branch (RGB) for the most massive stars. The input parameters for all the models are the same as for the $1.00 M_{\odot}$ model. In particular, we used the same initial lithium abundance for all masses. For solar mass and larger, we can neglect lithium depletion during the PMS, and the choice of the meteoritic lithium abundance since initial abundance of the models is straightforward. For lower masses than the solar one, initial lithium abundance should be lower since the lithium depletion during the PMS increases with decreasing mass. However, it is a tricky task to choose a value for the lithium abundance at the ZAMS as a function of stellar mass. D'Antona \& Mazzitelli (1994) determined that, depending on the physics input of convection treatment and opacities, a model with $0.8 M_{\odot}$ presents a lithium depleted of between 1.7 and $3.3 \mathrm{dex}$, at the age of $\alpha$ Persei. The smallest destruction $(\sim 1.7 \mathrm{dex})$ is the most consistent with lithium abundance observations of $\alpha$ Per and corresponds to models with the Kurucz (1991) opacities and Canuto \& Mazzitelli (1990) convection treatment. In this case, we chose to adopt the meteoritic lithium abundance as initial abundance as well, keeping in mind that, for these masses, it should be lower.

\section{Color-magnitude diagrams}

Accurate determinations of cluster ages and the masses of their members are an essential requirement when comparing our models to the cluster observations and to study the evolution with stellar mass of lithium abundance in each cluster. The classic way to obtain both is to compare model isochrones with data points on the color-magnitude diagram, as in Pace et al. (2012).

To construct the color-magnitude diagram of the open cluster Hyades presented in Fig. 1, we used a reddening $E(B-V)=$ $0.001 \mathrm{mag}$ (Taylor 2006), a distance modulus $(m-M)_{0}=$ 3.35 mag (McArthur et al. 2011; Perryman et al. 1998), and a metallicity $[\mathrm{Fe} / \mathrm{H}]=+0.13$ (Paulson et al. 2003). We plot the whole sample of Hyades stars with magnitudes from the HIPPARCOS catalogue along with our working sample of stars form Takeda et al. (2013) for which we have lithium abundance estimation. Blue diamonds represent the known binaries of this sample. For the isochrones, we computed two grids of models, as presented in Sect. 3. Matching the position of the turn-off hook of the isochrones with the left envelope of the observations, we estimated an age of 0.69 Gyr with the grid of standard models, and 0.79 Gyr with the grid of models with extra mixing, which falls right in between the classical estimation of $625 \mathrm{Myr}$ (Perryman et al. 1998) and that by Brandt \& Huang (2015) of 950 Myr. The data points that are found significantly far away from the isochrone could be due to photometric errors, nonmembers, or binaries. We can see that the known binaries are mostly off the isochrone. Also, a non-detected companion in a binary system would make the star appear more luminous and colder than it is, therefore a bias could exist in the CMD that moves some points up and/or to the left. However, in the case of the Hyades, this effect should be very small as the cluster has been very well studied.

The color-magnitude diagram for NGC 752 is presented in Fig. 2. We built it using the grids of models exposed in Sect. 3. Then, we shifted the resulting isochrone according to

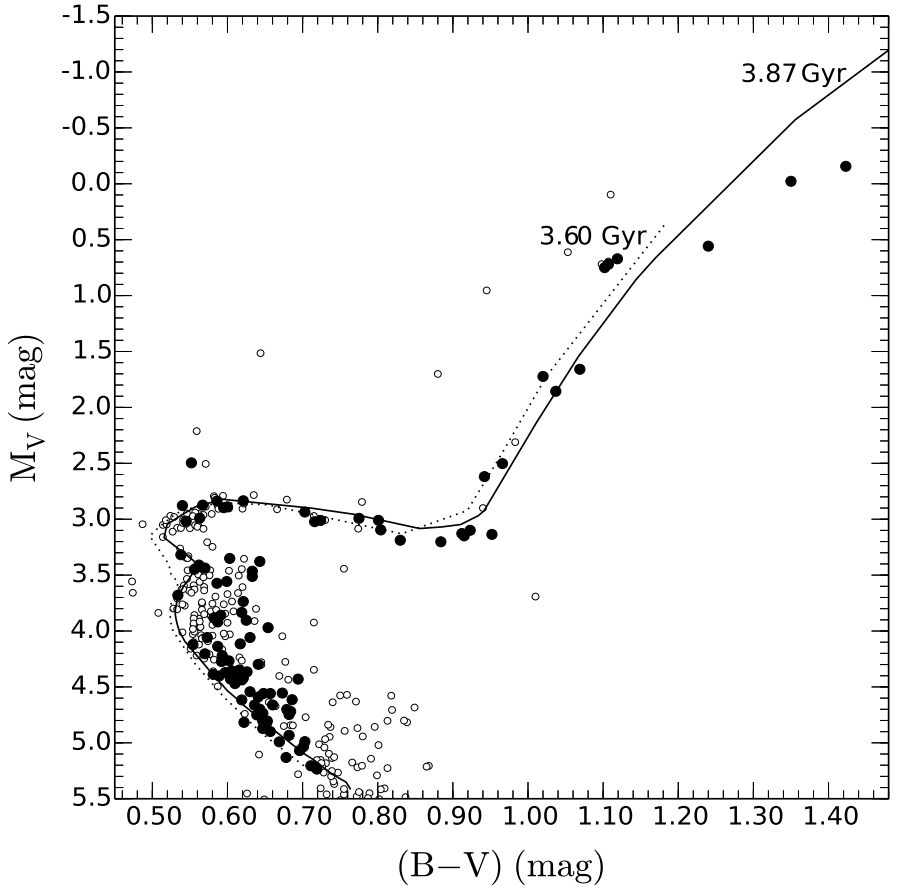

Fig. 3. Color-magnitude diagram of the open cluster M67. The black filled circles represent our work sample, i.e., stars with lithium abundance measurements. Stars with photometry available from Yadav et al. (2008), but with no lithium abundances, are displayed as open circles. The continuous isochrone from TGEC models with extra mixing corresponds to an age of $3.87 \mathrm{Gyr}$. The dotted line is the isochrone of 3.60 Gyr calculated from standard models.

a reddening of $E(B-V)=0.035 \mathrm{mag}$ and using a distance modulus of $(m-M)_{0}=8.25 \mathrm{mag}$ (Daniel et al. 1994). From the position of the turn-off hook of the isochrones, we estimated an age of $1.51 \mathrm{Gyr}$ with the grid of standard models, and $1.59 \mathrm{Gyr}$ with the grid of models with extra mixing. The positions of the turn-off hook and the giant branch of the isochrone depend on the input physics in the models such as metallicity, diffusion, and overshooting at the convective core. In particular, the cut-off of the diffusion when the model enters into the RGB or a bad calibration of the overshooting, could explain why the giants are off the isochrone.

For the open cluster, M 67, see the paper Pace et al. (2012), where model calibration and mass determination, a colormagnitude diagram, and an estimation of the cluster age of 3.87 Gyr have already been presented. In the present work, we added an isochrone that is made up of standard models to the color-magnitude diagram of M 67 and found an age of 3.60 Gyr. The new plot, including this isochrone, is presented in Fig. 3.

\section{Predicted lithium evolution as a function of mass and age}

In this section, we study the evolution of lithium abundance predicted by the models, as a function of mass in each cluster, and as a function of age, and we compare the evolution between the three clusters. In Fig. 4, we present the lithium abundance as a function of mass for our model isochrones with ages that are estimated previously for each cluster. Continuous lines are the isochrones from models with extra mixing, whereas dotted lines are the isochrones from standard models. We can identify several interesting facts in this figure. Models with mass lower than $\sim 1.10 M_{\odot}$, including rotation-induced mixing, seem 


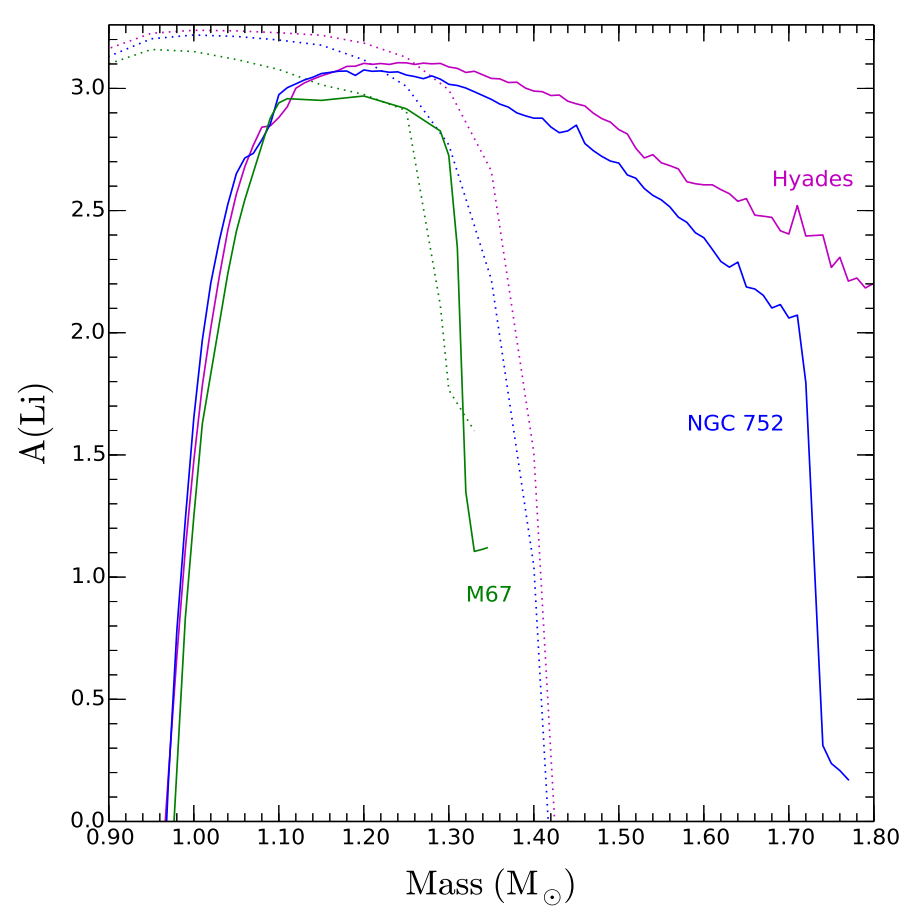

Fig. 4. Lithium abundance as a function of mass for isochrones with ages estimated for each cluster. Contiunuous isochrones are from models with extra mixing and dotted isohrones from standard models. Magenta lines represent the isochrones for the Hyades with $[\mathrm{Fe} / \mathrm{H}]=$ +0.13 and ages of $0.79 \mathrm{Gyr}$ and $0.69 \mathrm{Gyr}$, respectively; blue lines represent the isochrones for NGC 752 with $[\mathrm{Fe} / \mathrm{H}]=+0.00$ and ages of $1.59 \mathrm{Gyr}$ and $1.51 \mathrm{Gyr}$, respectively; and green lines represent the isochrones for $\mathrm{M} 67$ with $[\mathrm{Fe} / \mathrm{H}]=+0.01$ and ages of $3.87 \mathrm{Gyr}$ and $3.60 \mathrm{Gyr}$, respectively.

to destroy their lithium early in the MS, but depletion continues throughout the main sequence, with a lower rate after Hyades age though. For $1.00 M_{\odot}$, the lithium abundance difference between models of $1.59 \mathrm{Gyr}$ for NGC 752 and models of $3.87 \mathrm{Gyr}$ for M 67 is about 0.5 dex. In this mass range, which is characterized by the presence of an extensive outer convective zone, lithium destruction is strongly dependent on stellar mass. We notice that the lithium depletion predicted at the Hyades age is greater than at the age of NGC 752 owing to the difference of metallicity between the models of the Hyades and the models of NGC 752. Indeed, the Hyades members are much more metallic than the NGC 752 members, and thus the models of the former have a deeper convective zone than members of the latter (see Fig. 5), leading to a larger and quicker lithium depletion. The mixing that occurs below that convective zone is mainly driven by rotation during the PMS and the beginning of the MS. However, surface lithium destruction during the PMS is significant only for the latest-type stars, and stars with masses around the solar mass present a slight lithium depletion at the ZAMS Randich et al. (1997). In our models, PMS is not taken into account, but we calibrated our solar model to obtain the lithium abundance at the solar age, adopting the meteoritic lithium abundance of Asplund et al. (2009) as initial abundance. This implies that we probably used a stronger mixing than would have been necessary with a lower initial abundance owing to the slight depletion during the PMS. For lower masses, which should present a lower lithium abundance at the ZAMS, caused by the lithium destruction during the PMS, we expect lower lithium abundance than calculated by our models at the ages of the three clusters, and thus a steeper isochrone between 0.80 and $1.10 M_{\odot}$.

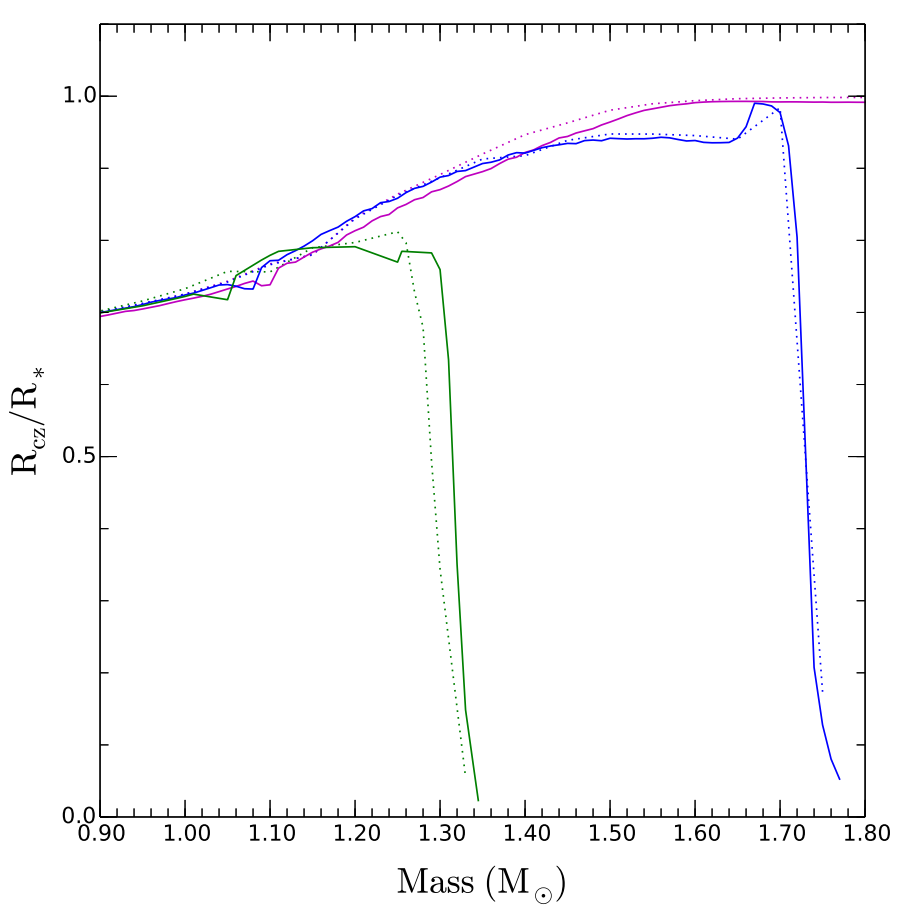

Fig. 5. Relative inner convective zone radius as a function of stellar mass for isochrones with ages estimated for each cluster. The line types and colors are the same as in Fig. 4.

For models with extra mixing with masses between 1.10 and $1.30 M_{\odot}$, we observe the presence of a lithium plateau where the element is barely destroyed during evolution in the MS and independently of stellar mass. In these stars, the outer convective zone is very shallow and the turbulence is weak. The rotationinduced mixing below the convective zone does not sink deep enough to bring the lithium to the destruction layers. For these masses, lithium destruction during the PMS is negligible and it is consistent to use the same initial lithium abundance as for the solar model. Furthermore, a decrease in the mixing efficiency should not have any significant effect. The lithium abundance on this plateau seems to slightly decrease with time, at a rate of about 0.2 dex between Hyades age and M 67 age.

When the mass increases, lithium abundance decreases with mass and age. This is due to the gravitational diffusion process below the convective zone. Lithium particles continuously sink below the convective zone owing to gravity and the amount of lithium in the convective zone decreases with time. For larger masses, the process is more efficient.

For the highest masses, we can see the dramatic decrease of lithium abundance with age due to the entry of the models in the red giant branch. When the star leaves the main sequence, the outer convective zone deepens during the first dredge-up and the lithium is diluted in lithium-free layers. In M 67, this occurs in stars with masses around $1.30 M_{\odot}$, although in NGC 752 this process can be observed for masses larger than $1.70 M_{\odot}$.

Standard models have a totaly different behavior. For masses lower than about $1.25 M_{\odot}$, depletion of lithium abundance is very weak and is due to microscopic diffusion below the convective zone. When the mass increases, the outer convective zone shrinks and the diffusion is more efficient with time. For larger masses, the convective zone is very thin (see Fig. 5) and microscopic diffusion takes place on a timescale much smaller than the stellar life time on the main sequence. Surface lithium abundance is depleted very quickly. From comparison with models 
with extra mixing, we can deduce that the rotation-induced mixing prevents the lithium depeletion from microscopic diffusion in this range of masses, until the first dredge-up. These statements are long-standing results (see Schatzman 1969).

Figure 5 shows the relative radius at the base of the convective zone along our three isochrones for the three open clusters and for the two sets of models as a function of stellar mass. The depth of the convection zone for the two sets of models is very similar, in contradiction with the completely different behavior of the lithium depletion. However, in the case of the models with meridional circulation, the extra mixing lies below the base of the convective zone, increasing greatly the depth of the chemical transport. For low-mass stars, the outer convective zone becomes shallower when the mass increases. For mass lower than $1.10 M_{\odot}$, this explains the mass-dependence of the lithium depletion. The convective zone of stars in the lithium plateau, i.e., between 1.10 and $1.30 M_{\odot}$, is not deep enough for mixing mechanisms to reach the layers in which lithium could be destroyed. For larger masses, in the cases of the Hyades $\left(M>1.60 M_{\odot}\right)$ and NGC $752\left(1.40 M_{\odot}<M<1.70 M_{\odot}\right)$, we can see that the slight slope in lithium depletion as a function of mass is not due to the depth of the convective zone, which remains nearly constant in this range of masses. As we explained, this lithium destruction is due to microscopic diffusion, which increases with mass and age. For larger masses, the dramatic decrease of lithium abundances corresponds to the strong deepening of the convective zone during the first dredge-up, at the beginning of the RGB. In the case of standard models, as explained above, the base of the convective zone is not deep enough to reach the lithium destruction layers, and the lithium depletion is controlled by the microscopic diffusion process, which is efficient for masses larger than about $1.25 M_{\odot}$

\section{Comparison of the predicted lithium abundance and observations}

For the Hyades, we estimated the mass for each star of our sample by picking the closest isochrone point to its data point on the color-magnitude diagram, and interpolating the value of the mass from the values for which the models were actually run. We used the isochrone calculated from models with extra mixing. Results of this interpolation are given in Table A.1. In Fig. 6, we present the lithium abundances of our sample as a function of the inferred stellar mass. We also plot the corresponding isochrones from both set of models. For stars with masses lower than $1.10 M_{\odot}$, lithium destruction occurs as soon as the evolution starts. Stars with these masses present low lithium abundance at the age of the Hyades. Our models with extra mixing reproduce the depletion as observed in Fig. 6. The models present a more severe depletion for this mass range. Standard models do not reproduce to any extent the lithium abundances of these stars.

For this cluster, the lithium plateau at $A(\mathrm{Li}) \sim 3.0$ for stars with masses larger than $1.10 M_{\odot}$ is also well reproduced. However, the Li-dip for stars with masses between 1.30 and $1.45 M_{\odot}$ is not reproduced by our models, thus suggesting that the mixing mechanism responsible for the Li-dip is not related with meridional circulation. However, it is interesting to note that the strong decrease of the lithium abundance in standard models corresponds to the Li-dip. For stars with masses larger than $1.60 \mathrm{M}_{\odot}$, there is a lack of lithium abundances estimation and we cannot give any formal comparison for the Hyades age. Known binaries have the same behavior as single stars.

For NGC 752, results of the interpolation are given in Table A.2. In Fig. 7, we plot the lithium abundances of our

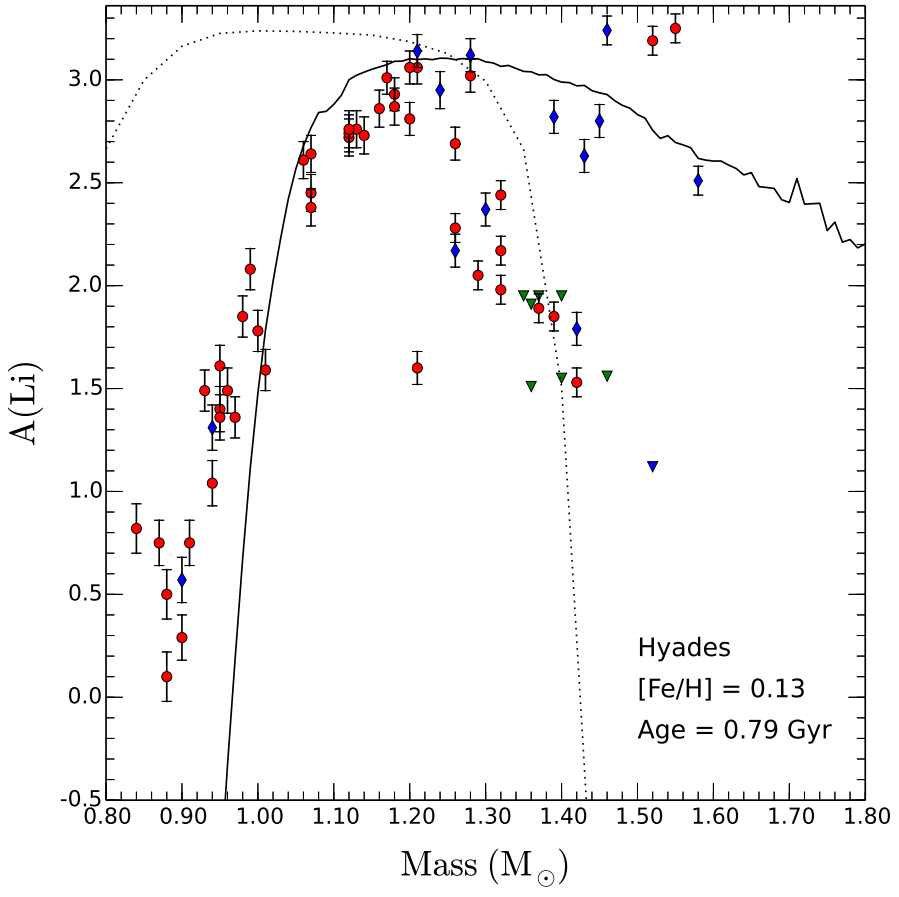

Fig. 6. Lithium abundances of our sample as a function of the inferred stellar mass for the Hyades. Red filled circles represent stars with the lithium abundance determined. Green triangles represent the lithium abundance upper limits. Blue diamond represent the known binaries. Continuous line corresponds to the lithium abundance predicted by models with rotation-induced mixing at the age of the Hyades, whereas the dotted line corresponds to the standard models.

sample as a function of the inferred stellar mass for this cluster. We also present the corresponding isochrones from our two sets of models, with the ages deduced from the color-magnitude diagram. As in the Hyades, we observe a good agreement of the models with rotation-induced mixing for the stars of NGC 752 with masses that are lower than $1.10 M_{\odot}$. However, for stars in the plateau, observed lithium abundances are lower by $\sim 0.3 \mathrm{dex}$ in relation to to the models' predictions. The Li-dip is still not reproduced. The models with masses larger than $1.60 M_{\odot}$ seem to deplete too much lithium. Again, standard models do not reproduce the lithium distribution of the NGC 752 cluster, and the decrease of lithium abundance for more massive stars corresponds to the Li-dip. The lithium abundances of binaries have no pecularity compared to single stars. This implies that the binarity has no influence on lithium depletion of these stars and thus, and that there is no tidal effect in the observed systems. It suggests that there are no close binaries, and that an analysis of the spectra of the two components of each system should be done in the future to know if they can be separated or not.

Figure 8 is the M 67 counterpart of Figs. 6 and 7. It is published by Pace et al. (2012) and shown here for better clarity and discussion. The results of the interpolation on the isochrone are also available in the online version of Pace et al. (2012). In this paper, the authors observed a good agreement between models and observations for stars with masses lower than $1.10 M_{\odot}$. The difference between the observed and the predicted plateaus is about 0.45 dex, showing an increase of this difference with age. The Li-dip, which lies around $1.34 M_{\odot}$ (Balachandran 1995), is not entirely reproduced in depth, the decrease of lithium abundance in the models is only due to the deepening of the outer convective zone, when the star enters in the RGB. Extra mixing mechanisms responsible for the Li-dip are not yet identified. 


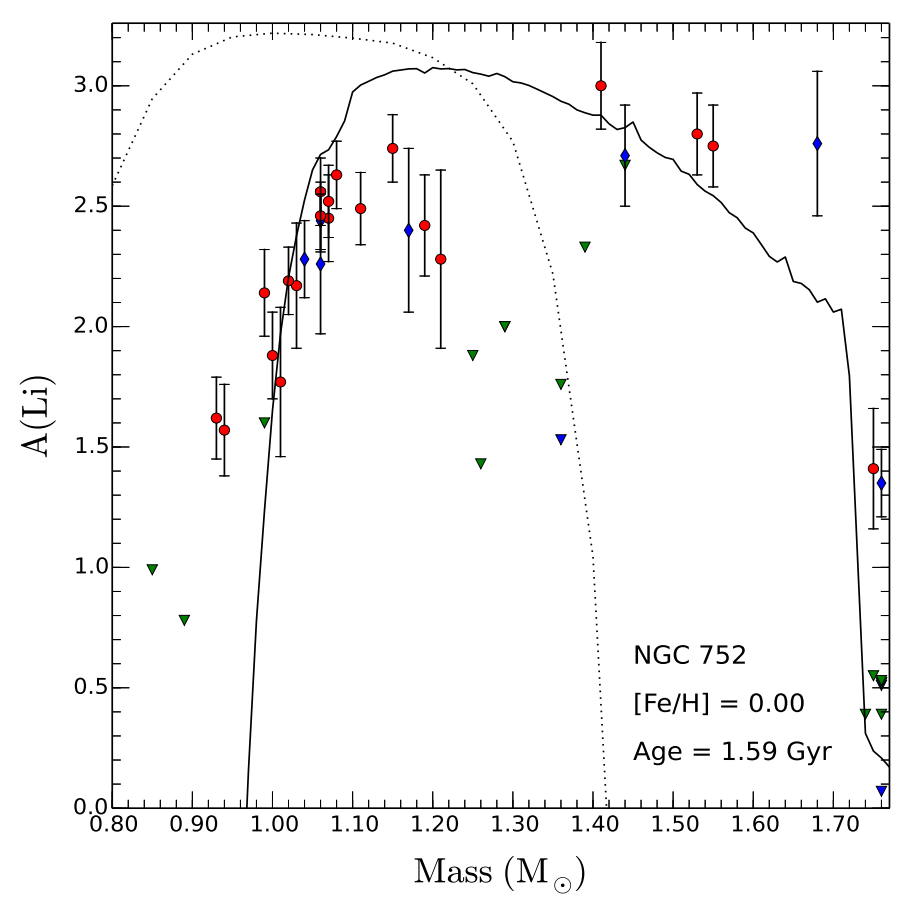

Fig. 7. Same as Fig. 6 but for the open cluster NGC 752.

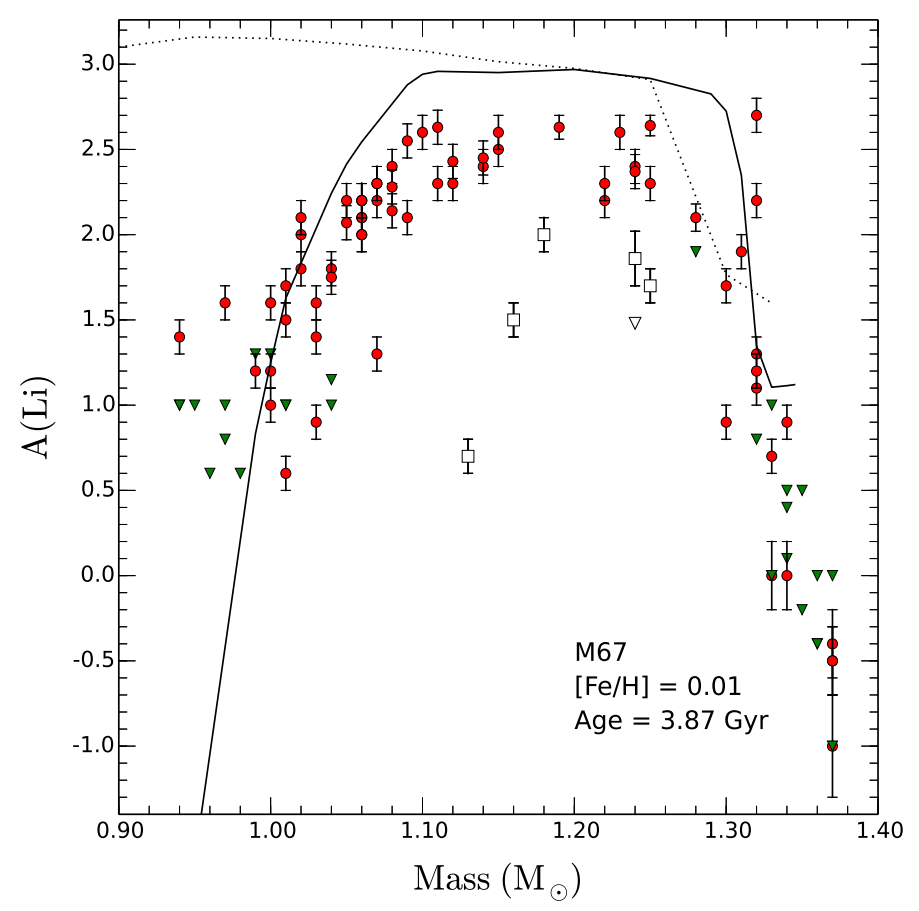

Fig. 8. Same as Figs. 6 and 7, but for the open cluster M67. The open squares represents the deviant stars as discussed in the Sect. 5.3 of Pace et al. (2012).

\section{Discussion}

Based on previous results with M 67 (Pace et al. 2012) and the new analysis for the Hyades and NGC 752, the calibration of evolution models on the atmospheric parameters of the Sun enables us to reproduce the qualitative distribution of lithium abundance as a function of the stellar mass for different ages. Lithium abundance is a crescent function of mass for $M<1.10 M_{\odot}$, where the depth of the outer convective zone plays a crucial role in the efficiency of the meridional circulation to bring lithium from the base of the convective zone to the destruction layers. As expected, solar-calibrated mixing is suitable for solar-type stars at all stages of the evolution. For lower masses, our mixing seems to deplete too much lithium, too soon, and this discrepancy would be even larger if lithium depletion during the PMS had been taken into account.

For higher masses, we observe a plateau between 1.10 and $1.30 M_{\odot}$ in the three clusters where lithium remains constant as a function of stellar mass. This plateau is reproduced by our models. For these masses the outer convective zone is too shallow to allow the meridional circulation to bring lithium particles to the destruction layers. The lithium abundance in this plateau decreases with time, due to microscopic diffusion below the convective zone. Indeed, in Fig. 4, we can see that standard models present nearly the same lithium abundances as models with rotation-induced mixing in this range of masses. However, our models fail to reproduce quantitatively this decrease and the difference between the observed and the predicted plateaus increases with age. Since the turbulence, which is due to meridional circulation, prevents lithium to diffuse below the convective zone, it can indicate that our parametrization leads to a too strong efficiency of the meridional circulation for theses masses.

In the three clusters, we observe a Li-dip, which lies around $1.34 M_{\odot}$. Our models with meridional circulation calibrated on the Sun do not reproduce this Li-dip. These results can be interpreted in different ways. As already stated, there is no a priori reason why the calibration of the parameters of the extra mixing for the solar model should also hold for different masses, and at different ages. It has been shown and discussed by Théado \& Vauclair (2003) and Pace et al. (2012) that it is possible to reproduce the Li-dip by calibrating the meridional circulation for each mass. We chose not to use this artificial parametric way and we focused on the study of the discrepancy in the solar mixing as a function of mass and its evolution. Furthermore, our results indicate that lithium depletion in these stars likely involves another mechanism besides meridional circulation. This other mechanism can be linked with angular momentum transport, like internal gravity waves (Talon \& Charbonnel 2005). Internal gravity waves (IGW) are produced and excited by stochastic movement at the base of the convective zone and they transport mainly negative angular momentum through the radiative zone. They are mostly efficient in low-mass stars. If we introduce IGW in a solar model, part of the angular momentum should be transported by these waves, reducing the efficiency of the meridional circulation. Thus, we would have to increase the turbulent diffusion coefficients to reproduce the solar lithium depletion. This increase could be enough to destroy lithium in greater masses, where IGW are much less efficient, and reproduce the decrease of the lithium abundance in the plateau with time and the Li-dip. These new physics need to be tested for different masses. Moreover, it cannot be a coincidence that, for the three clusters, the Li-dip corresponds to the masses for which microscopic diffusion becomes very efficient. A possible explanation could be that, for some reason, the rotation-induced turbulence, which prevents microscopic diffusion being efficient in this range of masses, becomes frozen.

We are also able to observe that for lower masses, the dispersion of lithium abundance seems to increase with age. The same trend is found for clusters studied by Randich (2008, 2009) for stars with a similar range of effective temperature. Figure 9 shows the cumulative distribution of lithium abundance for a shallow range of mass around the solar value (between 0.95 and $\left.1.05 M_{\odot}\right)$ in both open clusters with solar metallicity, NGC 752 


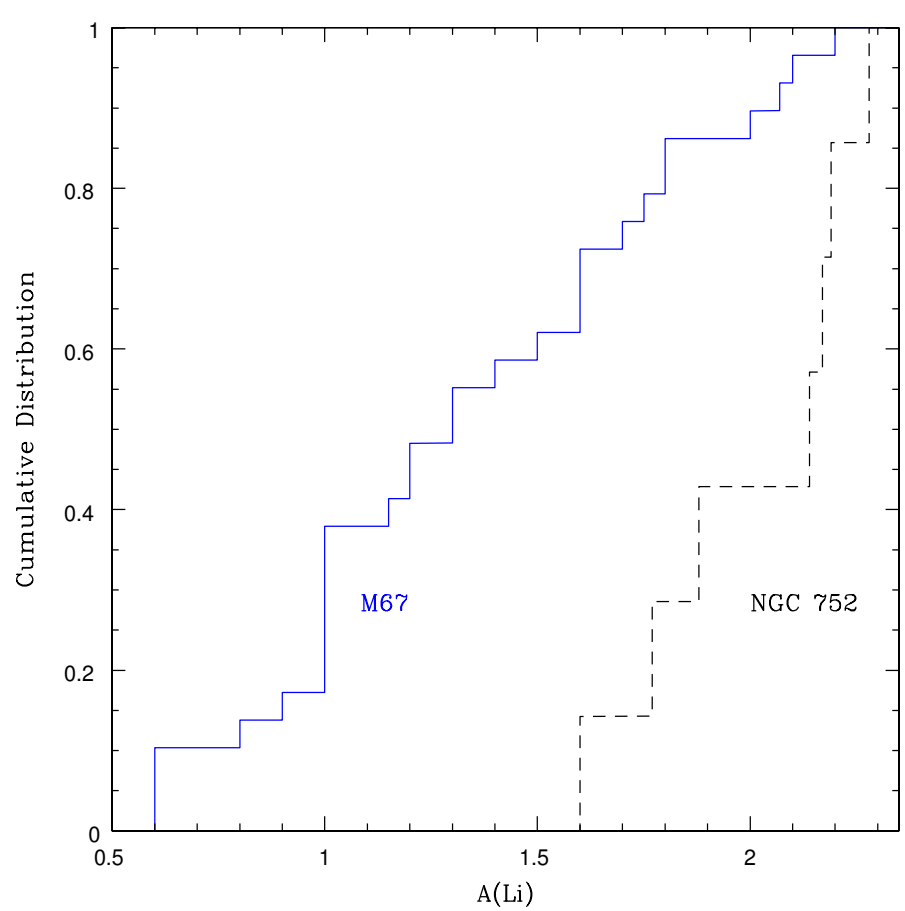

Fig. 9. Cumulative distribution for lithium abundance of NGC 752 (dotted line) stars and M 67 (continuous blue line) stars with a stellar mass between 0.95 and $1.05 M_{\odot}$.

and M 67. Since the Hyades is overmetallic, it would produce an additional bias in comparison. Indeed, a higher metallicity implies a deeper convective zone, and thus a larger efficiency of the transport mechanisms of lithium particles below the convective zone. In this figure, M 67 presents a dispersion in $A(\mathrm{Li})$ from 0.6 to 2.2 dex for masses between 0.95 and $1.05 M_{\odot}$. NGC 752 presents a lower dispersion from 1.6 to 2.3 dex in the same range of masses. The dispersion seems to increase with age between $1.59 \mathrm{Gyr}$ and 3.87 Gyr. However, the low number of stars with masses between 0.95 and $1.05 M_{\odot}$ for NGC 752 is not enough for a definitive conclusion about the evolution of the lithium abundance dispersion with age for solar-type stars. In Duarte et al. (in prep.), the authors show that the same dispersion exists for solar twin field stars. The authors use a sample of 88 solar twin stars from Ramírez et al. (2014) and do a comparative study of lithium abundance, rotation period, and magnetic activity as a function of stellar mass. Lithium abundance in solar twin field stars span a wide range of values, which cannot be explained by the small differences in mass. This range of dispersion for stars with estimated ages of between 2.0 and 5.0 Gyr is equivalent to the one we can see in the MS region in M $67\left(M \leqslant 1.10 M_{\odot}\right)$.

It will be important to understand this point in a near future, because it could be closely connected with the internal differential rotation evolution. Some studies put forward the idea that the dispersion in lithium abundances in solar-type stars is due to the different rotation history (see Charbonnel \& Talon 2005). The way that the magnetic field of the protostar is coupled with the accretion disk determines the magnetic brake that the star undergoes during the PMS, and the initial rotation rate of the star at the ZAMS (see Matt \& Pudritz 2005). Furthermore, stellar winds have a strong influence on rotation in the MS, removing angular momentum from the surface convective zone of the star. This increases the differential rotation between the convective zone and the radiative interior, and thus the efficency of the tachocline, which plays a major role on lithium destruction. As a result, the resulting decrease in the rotation rate causes significant changes in the wind strength (Johnstone et al. 2015).

This result indicates that lithium abundance is not such a good age indicator for solar-type stars, when used alone. Rotation period through gyrochronology (Barnes 2010) and asteroseismology must be taken into account to infer stellar age.

\section{Conclusions}

In this work, we have focused our study on the evolution of the atmospheric lithium abundance for open cluster, low-mass star members as a function of mass and age. We used three open clusters of different ages: the Hyades, with an estimated age of 0.79 Gyr, NGC 752, with an age of 1.59 Gyr, and M 67, with 3.87 Gyr. For each cluster, we show how strong the dependence of lithium abundance is with mass. Models that include meridional circulation reproduce most of the shape of lithium depletion. However, they fail to reproduce the Li-dip. Comparisons between clusters show that microscopic diffusion plays a role in F-type stars, where meridional circulation is inefficient in bringing lithium from the bottom of the shallow convective zone to the destruction layers. This study shows that there are two main reasons why using lithium abundance is tricky as an indicator of age. First, the lithium abundance shows a strong dependence on mass of a same age. The second reason is its dependency with transport mechanisms, which can be a function of rotation between others parameters. Comparisons of the rotation period distributions for solar-type stars in open clusters with different ages show that core and envelope rotations have to be coupled with a characteristic timescale of angular momentum transfer between them from a few Myr for the fastest rotators to nearly a hundred Myr for the slowest rotators (Denissenkov et al. 2010; Irwin et al. 2007). This angular momentum transfer seems to be mostly due to magnetic braking, but the interaction between meridional circulation and the differential rotation during this braking causes large turbulence which leads to a diffusion process of lithium from the base of the convective zone to the destruction layers. However, our models do not calculate this magnetic braking and the destruction of lithium is only driven by turbulence owing to classical meridional circulation. In the future, we intend to include the angular momentum redistribution in the stellar interior by magnetic braking in our models. Furthermore, in field stars one has to consider the intrinsic difficulty of getting accurate masses. This generates a greater dispersion and complicates the interpretation of the lithium abundance evolution as a function of age. However, for stars with very accurate mass determination, it is possible to use lithium abundance to diffentiate a young star from an old one. At the moment, we need observations of solar analog stars (with an effective temperature close to the solar one) of clusters with intermediate masses and solar metallicity to clarify the evolution of the dispersion of the lithium abundance in stars with solar mass.

Acknowledgements. This research made use of the SIMBAD database, operated at CDS, Strasbourg, France, and of WEBDA, an open cluster database developed and maintained by Jean-Claude Mermilliod. G.P. is supported by grant SFRH/BPD/39254/2007 and by the project PTDC/CTE-AST/098528/2008, funded by Fundação para a Ciência e a Tecnologia (FCT), Portugal. Research activities of the Stellar Board at the Federal University of Rio Grande do Norte are supported by continuous grants from CNPq and FAPERN Brazilian Agencies. J.D.N. and M.C. would like to acknowledge support from $\mathrm{CNPq}$ (Bolsa de Produtividade). 


\section{References}

Asplund, M., Grevesse, N., Sauval, A. J., \& Scott, P. 2009, ARA\&A, 47, 481 Bahcall, J. N., Pinsonneault, M. H., \& Wasserburg, G. J. 1995, Rev. Mod. Phys., 67,781

Balachandran, S. 1995, ApJ, 446, 203

Barnes, S. A. 2010, ApJ, 722, 222

Basu, S., \& Antia, H. M. 1997, MNRAS, 287, 189

Boesgaard, A. M. 1987, PASP, 99, 1067

Boesgaard, A. M., \& Budge, K. G. 1988, ApJ, 332, 410

Boesgaard, A. M., \& Tripicco, M. J. 1986, ApJ, 302, L49

Böhm-Vitense, E. 1958, Z. Astrophys., 46, 108

Brandt, T. D., \& Huang, C. X. 2015, ApJ, 807, 58

Brun, A. S., Turck-Chièze, S., Zahn, J.-P. 1998, in Structure and Dynamics of the Interior of the Sun and Sun-like Stars, ESA Publ. Div., 418, 439

Canuto, V. M., \& Mazzitelli, I. 1990, ApJ, 370, 295

Carrera, R., \& Pancino, E. 2011, A\&A, 535, A30

Casagrande, L., Ramírez, I., Meléndez, J., Bessell, M., \& Asplund, M. 2010, A\&A, 512, A54

Cayrel de Strobel, G. 1996, A\&ARv, 7, 243

Chapman, S. 1917, MNRAS, 77, 540

Chapman, S., \& Cowling, T. G. 1970, in The Mathematical Theory of NonUniform Gases, 3rd edn. (Cambridge University Press)

Charbonneau, P., Christensen-Dalsgaard, J., Henning, R. et al. 1999, ApJ, 527, 445

Charbonnel, C., \& Talon, S. 2005, Science, 309, 2189

Christensen-Dalsgaard, J., Dappen, W., Ajukov., S. V, et al. 1996, Science, 272, 1286

Cox, A. N., Guzik, J. A., \& Kidman, R. B. 1989, ApJ, 342, 1187

Daniel, S. A., Latham, D. W., Mathieu, R. D., \& Twarog, B. A. 1994, PASP, 106, 281

D’Antona, F., \& Mazzitelli, I. 1984, A\&A, 138, 431

D’Antona, F., \& Mazzitelli, I. 1994, ApJS, 90, 467

de Bruijne, J. H. J., Hoogerwerf, R., \& de Zeeuw, P. T. 2001, A\&A, 367, 111

Deliyannis, C. P., \& Pinsonneault, M. H. 1997, 488, 836

Denissenkov, P. A., Pinsonneault, M., Terndrup, D. M., \& Newsham, G. 2010, ApJ, 716, 1269

do Nascimento Jr., J.-D., Castro, M., Meléndez, J., et al. 2009, A\&A, 501, 687

Duncan, D. K., \& Jones, B. F. 1983, ApJ, 271, 663

Eddington, A. S. 1916, MNRAS, 77, 16

Eddington, A. S. 1926, The Internal Constitution of the Stars (1959 edn.: New York: Dover)

Garcia Lopez, R. J., Rebolo, R., \& Beckman, J. E. 1988, PASP, 100, 1489

Grevesse, N., \& Sauval, A. J. 1998, Space Sci. Rev., 85, 161

Heinemann, K. 1926, Astron. Nachr., 227, 193

Hobbs, L. M., \& Pilachowski, C. 1986a, ApJ, 309, L17

Hobbs, L. M., \& Pilachowski, C. 1986b, ApJ, 311, L37

Hobbs, L. M., \& Thorburn, J. A. 1992, AJ, 104, 669

Hui-Bon-Hoa, A. 2008, Ap\&SS, 316, 55

Irwin, J., Hodgkin, S., Aigrain, S., et al. 2007, MNRAS, 377, 741

Johnstone, C. P., Güdel, M., Brott, I., \& Lüftinger, T. 2015, A\&A, 577, A28

Jones, B. F., Fischer, D., Shetrone, M., \& Soderblom, D. R. 1997, AJ, 114, 352

Jones, B. F., Fischer, D., \& Soderblom, D. R. 1999, AJ, 117, 330

Kučinskas, A., Hauschildt, P. H., Ludwig, H.-G., et al. 2005, A\&A, 442, 281

Kurucz, R. L. 1991, in Stellar Atmospheres: Beyond the Classical Models, eds

L. Crivellari, I. Hubeny, \& D. G. Hummer, NATO ASI Ser. (Dordrecht: Kluwer), 441

Lind, K., Asplund, M., \& Barklem, P. S. 2009, A\&A, 503, 541

Maeder, A., \& Zahn, J.-P. 1998, A\&A, 334, 1000
Martin, E. L., Rebolo, R., Garcia Lopez, R. J., Magazzú, A., \& Pavlenko, Y. V. 1993, in IAU Colloq. 137, Inside the Stars, eds. A. Baglin, \& W. Weiss, ASP Conf. Ser., 40, 165

Matt, S. P., \& Pudritz, R. E. 2005, ApJ, 632, 135

McArthur, B. E., Benedict, G. F., Harrison, T. E., \& van Altena, W. 2011, AJ, 141,172

Meléndez, J., Ramírez, I., Casagrande, L., et al. 2010, Ap\&SS, 328, 193

Mestel, L., \& Moss, D. L. 1986, MNRAS, 221, 25

Michaud, G. 1970, ApJ, 160, 641

Pace, G., Castro, M., Meléndez, J., Théado, S., \& do Nascimento Jr., J.-D. 2012, A\&A, 541, A150

Paquette, C., Pelletier, C., Fontaine, G., \& Michaud, G. 1986, ApJS, 61, 177

Pasquini, L., Randich, S., \& Pallavicini, R. 1997, A\&A, 325, 535

Pasquini, L., Biazzo, K., Bonifacio, P., Randich, S., \& Bedin, L. R. 2008, A\&A, 489, 677

Paulson, D. B., Sneden, C., \& Cochran, W. D. 2003, AJ, 125, 3185

Perryman, M. A. C. 1997, Proc. ESA Symp. HiPPARCos - Venice 97, ESA SP, 402, 1

Perryman, M. A. C., Brown, A. G. A., Lebreton, Y., et al. 1998, A\&A, 331, 81

Pilachowski, C. A., \& Hobbs, L. M. 1988, PASP, 100, 336

Pilachowski, C., Saha, A., \& Hobbs, L. M. 1988, PASP, 100, 474

Pinsonneault, M. H. 1997, ARA\&A, 35, 557

Ramírez, I., Meléndez, J., Bean, J., et al. 2014, A\&A, 572, A48

Randich, S. 2008, Mem. Soc. Astron. It., 79, 516

Randich, S. 2009, in The Ages of Stars, eds. E. E. Mamajek, D. R. Soderblom, \& R. F. G. Wyse, IAU Symp. 258, 133

Randich, S., Aharpour, N., Pallavicini, R., Prosser, C. F., \& Stauffer, J. R. 1997, A\&A, 323, 86

Randich, S., Sestito, P., Primas, F., Pallavicini, R., \& Pasquini, L. 2006, A\&A, 450, 557

Randich, S., Primas, F., Pasquini, L., Sestito, P., \& Pallavicini, R. 2007, A\&A, 469, 163

Rebolo, R., \& Beckman, J. E. 1988, A\&A, 201, 267

Reddy, A. B. S., Giridhar, S., \& Lambert, D. L. 2012, MNRAS, 419, 1350

Richard, O., Vauclair, S., Charbonnel, C., \& Dziembowski, W.A. 1996, A\&A, 312,1000

Richard, O., Théado, S., \& Vauclair, S. 2004, Sol. Phys., 220, 243

Schatzman, E. 1969, A\&A, 3, 331

Schlattl, H. 2002, A\&A, 395, 85

Sestito, P., Randich, S., \& Pallavicini, R. 2004, A\&A, 426, 809

Soderblom, D. R., Oey, M. S., Johnson, D. R. H., \& Stone, R. P. S. 1990, AJ, 99, 595

Spiegel, E. A., \& Zahn, J.-P. 1992, A\&A, 265, 106

Spite, F., Spite, M., Peterson, R. C., \& Chaffee, F. H., Jr. 1987, A\&A, 171, L8

Sweet, P. A. 1950, MNRAS, 110, 548

Takeda, Y., Honda, S., Ohnishi, T., et al. 2013, PASJ, 65, 53

Talon, S., \& Charbonnel, C. 2005, A\&A, 440, 981

Talon, S., \& Zahn, J.-P. 1997, A\&A, 317, 749

Taylor, B. J. 2006, AJ, 132, 2453

Théado, S., \& Vauclair, S. 2003, ApJ, 587, 795

Thorburn, J. A., Hobbs, L. M., Deliyannis, C. P., \& Pinsonneault, M. H. 1993, ApJ, 415, 150

Turcotte, S. Richer, J., Michaud, G., Iglesias, C. A., \& Rogers, F. J. 1998, ApJ, 504, 539

van Leeuwen, F. 2007, A\&A, 474, 653

Vauclair, S., \& Théado, S. 2003, ApJ, 587, 777

Ventura, P., Zeppieri, A., Mazzitelli, I., \& D’Antona, F. 1998, A\&A, 331, 1011

Yadav, R. K. S., Bedin, L. R., Piotto, G., et al. 2008, A\&A, 484, 609

Zahn, J.-P. 1992, A\&A, 265, 115 


\section{Appendix A: Tables}

Table A.1. Results of the interpolation with the isochrone in the CMD for the Hyades star sample.

\begin{tabular}{|c|c|c|c|c|c|c|c|c|}
\hline ID & $\begin{array}{l}T_{\text {eff }} \\
(\mathrm{K})\end{array}$ & $\begin{array}{l}T_{\text {iso }} \\
(\mathrm{K})\end{array}$ & $\log g$ & $(\log g)_{\text {iso }}$ & $\begin{array}{c}\text { Mass } \\
M_{\odot}\end{array}$ & $\begin{array}{l}A(\mathrm{Li}) \\
(\mathrm{dex})\end{array}$ & $\begin{array}{c}\mathrm{d} A(\mathrm{Li}) \\
(\mathrm{dex})\end{array}$ & Binarity \\
\hline 24357 & 6934 & 7000 & 4.30 & 4.30 & 1.52 & 3.19 & 0.07 & $\mathrm{n}$ \\
\hline 26462 & 6971 & 7014 & 4.30 & 4.19 & 1.55 & 3.25 & 0.07 & $\mathrm{n}$ \\
\hline 26015 & 6795 & 7079 & 4.32 & 4.18 & 1.58 & 2.51 & 0.07 & $\mathrm{y}$ \\
\hline 26911 & 6783 & 6823 & 4.32 & 4.24 & 1.46 & 3.24 & 0.07 & $\mathrm{y}$ \\
\hline 27561 & 6728 & 6823 & 4.33 & 4.24 & 1.46 & $<1.56$ & & $\mathrm{n}$ \\
\hline 18404 & 6714 & 6668 & 4.33 & 4.26 & 1.40 & $<1.55$ & & $\mathrm{n}$ \\
\hline 25102 & 6705 & 6606 & 4.33 & 4.28 & 1.37 & $<1.95$ & & $\mathrm{n}$ \\
\hline 28736 & 6693 & 6714 & 4.33 & 4.25 & 1.42 & 1.53 & 0.07 & $\mathrm{n}$ \\
\hline 26345 & 6660 & 6591 & 4.34 & 4.29 & 1.36 & $<1.51$ & & $\mathrm{n}$ \\
\hline 28568 & 6656 & 6591 & 4.34 & 4.29 & 1.36 & $<1.91$ & & $\mathrm{n}$ \\
\hline 28911 & 6651 & 6606 & 4.34 & 4.28 & 1.37 & 1.89 & 0.07 & $\mathrm{n}$ \\
\hline 27534 & 6598 & 6668 & 4.34 & 4.26 & 1.40 & $<1.95$ & & $\mathrm{n}$ \\
\hline 29225 & 6593 & 6561 & 4.35 & 4.29 & 1.35 & $<1.95$ & & $\mathrm{n}$ \\
\hline 27848 & 6558 & 6652 & 4.35 & 4.27 & 1.39 & 1.85 & 0.07 & $\mathrm{n}$ \\
\hline 31845 & 6558 & 6501 & 4.35 & 4.31 & 1.32 & 2.17 & 0.07 & $\mathrm{n}$ \\
\hline 28406 & 6554 & 6501 & 4.35 & 4.31 & 1.32 & 2.44 & 0.07 & $\mathrm{n}$ \\
\hline 27483 & 6533 & 6934 & 4.36 & 4.20 & 1.52 & $<1.12$ & & $\mathrm{y}$ \\
\hline 27731 & 6507 & 6426 & 4.36 & 4.33 & 1.29 & 2.05 & 0.07 & $\mathrm{n}$ \\
\hline 28483 & 6474 & 6501 & 4.37 & 4.31 & 1.32 & 1.98 & 0.07 & $\mathrm{n}$ \\
\hline 28608 & 6465 & 6353 & 4.37 & 4.35 & 1.26 & 2.28 & 0.07 & $\mathrm{n}$ \\
\hline 30869 & 6339 & 6714 & 4.39 & 4.25 & 1.42 & 1.79 & 0.08 & $\mathrm{y}$ \\
\hline 27383 & 6310 & 6441 & 4.40 & 4.33 & 1.30 & 2.37 & 0.08 & $\mathrm{y}$ \\
\hline 27991 & 6310 & 6792 & 4.40 & 4.24 & 1.45 & 2.80 & 0.08 & $\mathrm{y}$ \\
\hline 26784 & 6291 & 6397 & 4.40 & 4.34 & 1.28 & 3.02 & 0.08 & $\mathrm{n}$ \\
\hline 27808 & 6275 & 6223 & 4.41 & 4.39 & 1.21 & 3.06 & 0.08 & $\mathrm{n}$ \\
\hline 28394 & 6242 & 6353 & 4.41 & 4.35 & 1.26 & 2.17 & 0.08 & $\mathrm{y}$ \\
\hline 30809 & 6239 & 6223 & 4.41 & 4.39 & 1.21 & 1.60 & 0.08 & $\mathrm{n}$ \\
\hline 28363 & 6202 & 6745 & 4.42 & 4.25 & 1.43 & 2.63 & 0.08 & $\mathrm{y}$ \\
\hline 30738 & 6202 & 6397 & 4.42 & 4.34 & 1.28 & 3.12 & 0.08 & $\mathrm{y}$ \\
\hline 28205 & 6199 & 6194 & 4.42 & 4.40 & 1.20 & 3.06 & 0.08 & $\mathrm{n}$ \\
\hline 28635 & 6186 & 6123 & 4.42 & 4.42 & 1.17 & 3.01 & 0.08 & $\mathrm{n}$ \\
\hline 30810 & 6174 & 6652 & 4.43 & 4.27 & 1.39 & 2.82 & 0.08 & $\mathrm{y}$ \\
\hline 35768 & 6122 & 6353 & 4.44 & 4.35 & 1.26 & 2.69 & 0.08 & $\mathrm{n}$ \\
\hline 28033 & 6118 & 6223 & 4.44 & 4.39 & 1.21 & 3.14 & 0.08 & $\mathrm{y}$ \\
\hline 27406 & 6107 & 6137 & 4.44 & 4.41 & 1.18 & 2.93 & 0.08 & $\mathrm{n}$ \\
\hline 28237 & 6107 & 6194 & 4.44 & 4.40 & 1.20 & 2.81 & 0.08 & $\mathrm{n}$ \\
\hline 20430 & 6079 & 6309 & 4.45 & 4.37 & 1.24 & 2.95 & 0.09 & $\mathrm{y}$ \\
\hline 14127 & 6079 & 5984 & 4.45 & 4.46 & 1.12 & 2.72 & 0.09 & $\mathrm{n}$ \\
\hline 29419 & 6045 & 6095 & 4.45 & 4.43 & 1.16 & 2.86 & 0.09 & $\mathrm{n}$ \\
\hline 30589 & 6037 & 6137 & 4.45 & 4.41 & 1.18 & 2.87 & 0.09 & $\mathrm{n}$ \\
\hline 25825 & 5983 & 5984 & 4.46 & 4.46 & 1.12 & 2.74 & 0.09 & $\mathrm{n}$ \\
\hline 27859 & 5961 & 6039 & 4.47 & 4.44 & 1.14 & 2.73 & 0.09 & $\mathrm{n}$ \\
\hline 28344 & 5924 & 6011 & 4.47 & 4.45 & 1.13 & 2.76 & 0.09 & $\mathrm{n}$ \\
\hline 20439 & 5894 & 5984 & 4.48 & 4.46 & 1.12 & 2.76 & 0.09 & $\mathrm{n}$ \\
\hline 28992 & 5844 & 5834 & 4.49 & 4.49 & 1.07 & 2.64 & 0.09 & $\mathrm{n}$ \\
\hline 26767 & 5812 & 5794 & 4.50 & 4.50 & 1.06 & 2.61 & 0.09 & $\mathrm{n}$ \\
\hline 26736 & 5757 & 5834 & 4.51 & 4.49 & 1.07 & 2.45 & 0.09 & $\mathrm{n}$ \\
\hline 28099 & 5735 & 5834 & 4.51 & 4.49 & 1.07 & 2.38 & 0.09 & $\mathrm{n}$ \\
\hline 26756 & 5640 & 5571 & 4.53 & 4.55 & 0.99 & 2.08 & 0.10 & $\mathrm{n}$ \\
\hline 27282 & 5553 & 5610 & 4.54 & 4.54 & 1.00 & 1.78 & 0.10 & $\mathrm{n}$ \\
\hline 240648 & 5527 & 5533 & 4.54 & 4.55 & 0.98 & 1.85 & 0.10 & $\mathrm{n}$ \\
\hline 19902 & 5522 & 5636 & 4.55 & 4.53 & 1.01 & 1.59 & 0.10 & $\mathrm{n}$ \\
\hline 28593 & 5516 & 5508 & 4.55 & 4.56 & 0.97 & 1.36 & 0.10 & $\mathrm{n}$ \\
\hline
\end{tabular}

Notes. First column: star ID, second column: effective temperature, third column: effective temperature of the closest point in the isochrone, fourth column: $\log g$, fifth column: $\log g$ of the closest point in the isochrone, sixth column: mass of the closest point in the isochrone, seventh column: lithium abundance, eighth column: lithium abundance error corresponding to an uncertainty of $100 \mathrm{~K}$ in $T_{\text {eff }}$, ninth column: star belonging (y) or not (n) to a binary system. $T_{\text {eff }}(\mathrm{Col} .2), \log g$ (Col. 4), and the non-LTE lithium abundance $A(\mathrm{Li})(\mathrm{Col} .7)$ are from Takeda et al. (2013). 
A\&A 590, A94 (2016)

Table A.1. continued.

\begin{tabular}{ccccccccc}
\hline \hline ID & $\begin{array}{c}T_{\text {eff }} \\
(\mathrm{K})\end{array}$ & $\begin{array}{c}T_{\text {iso }} \\
(\mathrm{K})\end{array}$ & $\log g$ & $(\log g)_{\text {iso }}$ & $\begin{array}{c}\text { Mass } \\
M_{\odot}\end{array}$ & $\begin{array}{c}A(\mathrm{Li}) \\
(\mathrm{dex})\end{array}$ & $\begin{array}{c}\mathrm{d} A(\mathrm{Li}) \\
(\mathrm{dex})\end{array}$ & Binarity \\
\hline 31609 & 5508 & 5432 & 4.55 & 4.57 & 0.95 & 1.61 & 0.10 & $\mathrm{n}$ \\
27250 & 5485 & 5357 & 4.55 & 4.58 & 0.93 & 1.49 & 0.10 & $\mathrm{n}$ \\
27732 & 5449 & 5395 & 4.55 & 4.58 & 0.94 & 1.31 & 0.11 & $\mathrm{y}$ \\
32347 & 5429 & 5470 & 4.56 & 4.57 & 0.96 & 1.49 & 0.11 & $\mathrm{n}$ \\
242780 & 5429 & 5432 & 4.56 & 4.57 & 0.95 & 1.40 & 0.11 & $\mathrm{n}$ \\
283704 & 5426 & 5432 & 4.56 & 4.57 & 0.95 & 1.36 & 0.11 & $\mathrm{n}$ \\
284574 & 5303 & 5395 & 4.57 & 4.58 & 0.94 & 1.04 & 0.11 & $\mathrm{n}$ \\
284253 & 5297 & 5272 & 4.57 & 4.59 & 0.91 & 0.75 & 0.11 & $\mathrm{n}$ \\
285773 & 5254 & 5116 & 4.58 & 4.62 & 0.87 & 0.75 & 0.11 & $\mathrm{n}$ \\
30505 & 5249 & 5236 & 4.58 & 4.60 & 0.90 & 0.57 & 0.11 & $\mathrm{y}$ \\
28258 & 5235 & 5236 & 4.58 & 4.60 & 0.90 & 0.29 & 0.11 & $\mathrm{n}$ \\
27771 & 5196 & 5164 & 4.58 & 4.61 & 0.88 & 0.50 & 0.12 & $\mathrm{n}$ \\
28462 & 5172 & 4988 & 4.58 & 4.63 & 0.84 & 0.82 & 0.12 & $\mathrm{n}$ \\
285367 & 5114 & 5164 & 4.59 & 4.61 & 0.88 & 0.10 & 0.12 & $\mathrm{n}$ \\
\hline
\end{tabular}


M. Castro et al.: Mass effect on the lithium abundance evolution of open clusters

Table A.2. Results of the interpolation with the isochrone in the CMD for the NGC 752 star sample.

\begin{tabular}{|c|c|c|c|c|c|c|c|c|c|}
\hline ID & $\begin{array}{l}T_{\text {iso }} \\
(\mathrm{K})\end{array}$ & $(\log g)_{\text {iso }}$ & $\begin{array}{c}T_{\text {phot }} \\
(\mathrm{K})\end{array}$ & $\begin{array}{c}\text { Mass } \\
M_{\odot}\end{array}$ & $\begin{array}{l}E W_{\mathrm{Li}} \\
(\mathrm{m} \AA)\end{array}$ & $\begin{array}{c}\mathrm{d} E W_{\mathrm{Li}} \\
(\mathrm{m} \AA)\end{array}$ & $\begin{array}{l}A(\mathrm{Li}) \\
(\mathrm{dex})\end{array}$ & $\begin{array}{c}\mathrm{d} A(\mathrm{Li}) \\
(\mathrm{dex})\end{array}$ & Binarity \\
\hline $\mathrm{H} 10$ & 6854 & 3.82 & 6463 & 1.68 & 35 & 10 & 2.69 & 0.30 & $\mathrm{y}$ \\
\hline H88 & 6501 & 4.29 & 6195 & 1.29 & $<10$ & & $<2.00$ & & $\mathrm{n}$ \\
\hline H94 & 5308 & 4.59 & 5333 & 0.89 & $<5$ & & $<0.78$ & & $\mathrm{n}$ \\
\hline H123 & 6668 & 4.14 & 6523 & 1.44 & $<34$ & & $<2.67$ & & $\mathrm{n}$ \\
\hline H139 & 6501 & 4.29 & 6294 & 1.29 & $<10$ & & $<2.00$ & & $\mathrm{n}$ \\
\hline H185 & 6266 & 4.36 & 6030 & 1.19 & 42 & 10 & 2.31 & 0.21 & $\mathrm{n}$ \\
\hline H189 & 6668 & 4.17 & 6416 & 1.41 & 65 & 10 & 3.00 & 0.18 & $\mathrm{n}$ \\
\hline H222 & 6652 & 4.03 & 6555 & 1.55 & 45 & 10 & 2.68 & 0.17 & $\mathrm{n}$ \\
\hline $\mathrm{H} 227$ & 5116 & 4.62 & 4917 & 0.85 & $<12$ & & $<0.99$ & & $\mathrm{n}$ \\
\hline H235 & 6606 & 4.21 & 6203 & 1.36 & $<3$ & & $<1.53$ & & $\mathrm{y}$ \\
\hline H237 & 6223 & 4.38 & 5797 & 1.17 & 43 & 10 & 2.29 & 0.34 & $\mathrm{y}$ \\
\hline $\mathrm{H} 254$ & 6668 & 4.05 & 6617 & 1.53 & 49 & 10 & 2.74 & 0.17 & $\mathrm{n}$ \\
\hline H259 & 6637 & 4.19 & 6432 & 1.39 & $<17$ & & $<2.33$ & & $\mathrm{n}$ \\
\hline H265 & 5688 & 4.53 & 5614 & 0.99 & $<15$ & & $<1.60$ & & $\mathrm{n}$ \\
\hline H266 & 6668 & 4.15 & 6412 & 1.44 & 42 & 10 & 2.64 & 0.21 & $\mathrm{y}$ \\
\hline H293 & 6412 & 4.31 & 6188 & 1.25 & $<15$ & & $<1.32$ & & $\mathrm{n}$ \\
\hline H302 & 6606 & 4.22 & 6440 & 1.36 & $<5$ & & $<1.76$ & & $\mathrm{n}$ \\
\hline H304 & 6441 & 4.30 & 6199 & 1.26 & $<3$ & & $<1.43$ & & $\mathrm{n}$ \\
\hline PLA475 & 5929 & 4.47 & 5722 & 1.07 & 59 & 10 & 2.45 & 0.18 & $\mathrm{n}$ \\
\hline PLA520 & 5929 & 4.47 & 5893 & 1.07 & 67 & 12 & 2.52 & 0.15 & $\mathrm{n}$ \\
\hline PLA552 & 5888 & 4.48 & 5857 & 1.06 & 61 & 7 & 2.44 & 0.13 & $\mathrm{y}$ \\
\hline PLA648 & 6324 & 4.35 & 5844 & 1.21 & 24 & 8 & 2.28 & 0.37 & $\mathrm{n}$ \\
\hline PLA699 & 5847 & 4.50 & 5713 & 1.04 & 48 & 10 & 2.28 & 0.16 & y \\
\hline PLA701 & 5794 & 4.50 & 5526 & 1.03 & 42 & 12 & 2.17 & 0.26 & $\mathrm{n}$ \\
\hline PLA786 & 5727 & 4.52 & 5414 & 1.01 & 20 & 7 & 1.77 & 0.31 & $\mathrm{n}$ \\
\hline PLA859 & 5714 & 4.52 & 5584 & 1.00 & 26 & 7 & 1.88 & 0.18 & $\mathrm{n}$ \\
\hline PLA864 & 5902 & 4.47 & 5867 & 1.06 & 75 & 9 & 2.56 & 0.14 & $\mathrm{n}$ \\
\hline PLA889 & 5970 & 4.46 & 5956 & 1.08 & 78 & 11 & 2.63 & 0.14 & $\mathrm{n}$ \\
\hline PLA921 & 6053 & 4.44 & 5949 & 1.11 & 54 & 9 & 2.49 & 0.15 & $\mathrm{n}$ \\
\hline PLA964 & 5888 & 4.48 & 5854 & 1.06 & 64 & 9 & 2.46 & 0.14 & $\mathrm{n}$ \\
\hline PLA983 & 5767 & 4.51 & 5765 & 1.02 & 45 & 7 & 2.19 & 0.14 & $\mathrm{n}$ \\
\hline PLA993 & 5482 & 4.57 & 5475 & 0.94 & 20 & 6 & 1.57 & 0.19 & $\mathrm{n}$ \\
\hline PLA1012 & 6165 & 4.40 & 5996 & 1.15 & 74 & 10 & 2.74 & 0.14 & $\mathrm{n}$ \\
\hline PLA1107 & 5445 & 4.58 & 5614 & 0.93 & 24 & 5 & 1.62 & 0.17 & $\mathrm{n}$ \\
\hline PLA1284 & 5902 & 4.48 & 5564 & 1.06 & 42 & 9 & 2.26 & 0.29 & $\mathrm{y}$ \\
\hline PLA1365 & 5662 & 4.54 & 5470 & 0.99 & 48 & 8 & 2.14 & 0.18 & $\mathrm{n}$ \\
\hline H77 & 4764 & 2.93 & 4580 & 1.75 & $59 *$ & 10 & 1.41 & 0.25 & $\mathrm{n}$ \\
\hline H311 & 4677 & 2.75 & 4718 & 1.76 & $<12 *$ & & $<0.52$ & & $\mathrm{n}$ \\
\hline H137 & 4634 & 2.68 & 4773 & 1.76 & $<10^{*}$ & & $<0.39$ & & $\mathrm{n}$ \\
\hline H208 & 4623 & 2.65 & 4682 & 1.76 & $70 *$ & 10 & 1.35 & 0.14 & $\mathrm{y}$ \\
\hline $\mathrm{H} 213$ & 4666 & 2.73 & 4812 & 1.76 & $<12^{*}$ & & $<0.51$ & & $\mathrm{y}$ \\
\hline H1 & 4764 & 2.93 & 4934 & 1.75 & $<10 *$ & & $<0.55$ & & $\mathrm{n}$ \\
\hline H295 & 4731 & 2.87 & 4891 & 1.76 & $<10^{*}$ & & $<0.52$ & & $\mathrm{n}$ \\
\hline H186 & 4931 & 3.30 & 4891 & 1.74 & $<5^{*}$ & & $<0.39$ & & $\mathrm{n}$ \\
\hline H110 & 4645 & 2.70 & 5153 & 1.76 & $<5^{*}$ & & $<0.07$ & & $\mathrm{y}$ \\
\hline H24 & 4645 & 2.69 & 4812 & 1.76 & $<13^{*}$ & & $<0.53$ & & $\mathrm{n}$ \\
\hline
\end{tabular}

Notes. First column: star ID, second column: effective temperature of the closest point in the isochrone, third column: $\log g$ of the closest point in the isochrone, fourth column: photometric temperature, fifth column: mass of the closest point in the isochrone, sixth column: equivalent width of the Li line at $6708 \AA$, seventh column: equivalent width error, eighth column: lithium abundance, ninth column: lithium abundance error, tenth column: star belonging (y) or not (n) to a binary system. EW measurements derived from published lithium abundances are flagged with an asterisk. 